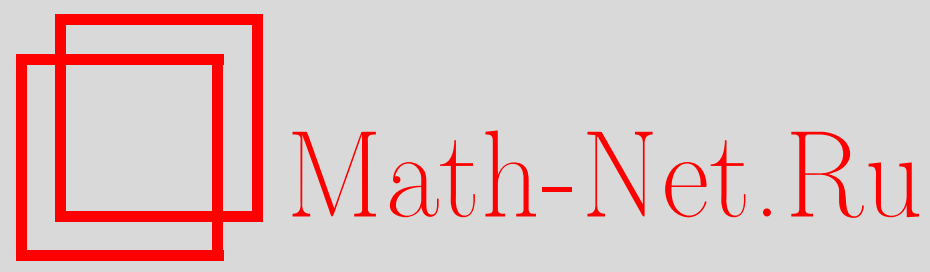

B. K. Карташов, Независимые системы порождающих и свойство Хопфа для унарных алгебр, Дискрет. матем., 2008, том 20, выпуск 4, 79-84

DOI: https://doi.org/10.4213/dm1027

Использование Общероссийского математического портала Math-Net.Ru подразумевает, что вы прочитали и согласны с пользовательским соглашением http://www . mathnet.ru/rus/agreement

Параметры загрузки:

IP: 35.173 .137 .237

26 апреля 2023 г., 12:20:00 


\title{
Независимые системы порождающих и свойство Хопфа для унарных алгебр
}

\author{
(c) 2008 г. $\quad$ В. К. Карташов
}

\begin{abstract}
Вводится понятие независимого множества элементов унарной алгебры как подмножества ее носителя, в котором каждый из любых двух элементов не принадлежит подалгебре, порожденной другим. Установлено, что любые две независимые системы порождающих унарной алгебры имеют одну и ту же мощность. С использованием этого утверждения доказано, что любая конечно порожденная унарная алгебра с коммутирующими между собой операциями обладает свойством Хопфа: каждый ее эпиэндоморфизм является автоморфизмом.
\end{abstract}

\section{1. Определения и вспомогательные результаты}

Настоящая работа содержит доказательство результатов, анонсированных автором в [1].

Далее $\mathbf{N}$ означает множество положительных целых чисел и $\mathbf{N}_{0}=\mathbf{N} \cup\{0\}$. Пусть $\mathfrak{U}=\langle A, \Omega\rangle-$ произвольная унарная алгебра. Через $\Omega^{*}$ обозначается моноид слов с порождающим множеством $\Omega$ относительно композиции. Единицей в $\Omega^{*}$ служит пустое слово $\varnothing$.

Результат $w(a)$ применения слова $w \in \Omega^{*}$ к элементу $a \in A$ определяется индуктивно. По определению полагаем $f^{0}(a)=\varnothing a=a, f^{n}(a)=f\left(f^{n-1}(a)\right)$ для произвольных $f \in \Omega, a \in A$.

Отсюда, если $w=w_{1} w_{2}$, то $w(a)=w_{1}\left(w_{2}(a)\right)$, где $w, w_{1}, w_{2} \in \Omega^{*}$ и $a \in A$. В дальнейшем, как правило, вместо $w(a)$ будем писать $w a$.

Далее для любого $a \in A$ через ( $a$ ) обозначается подалгебра алгебры $\mathfrak{A}$, порожденная элементом $a$.

Элементы $a, b \in A$ называются взаимно достижимыми (см. с. 66 в [2]), если $(a)=(b)$.

Очевидно, что отношение взаимной достижимости является эквивалентностью на множестве $A$. В дальнейшем ради краткости в случае, если элементы $a$ и $b$ находятся в этом отношении, ограничимся фразой, что элементы $a$ и $b$ эквивалентны, и записью $a \sim b$.

Класс эквивалентности $\sim$ с порождающим элементом $a$ называется слоем этого элемента и обозначается $S(a)$.

Элемент $a \in A$ называется выводимым, если $a=f(b)$ для некоторых $f \in \Omega$ и $b \in A$, и невыводимым в противном случае.

Будем говорить, что $a$ - базисный элемент, если подалгебра $(a)$, порожденная им, максимальна по включению в множестве всех однопорожденных подалгебр алгебры $\mathfrak{A}$, то есть

$$
(\forall b \in A)((a) \subseteq(b) \Rightarrow(a)=(b)) .
$$


Лемма 1. Пусть элемент а $\in$ А обладает одним из следующих свойств: выводимый, невыводимый, базисный. Тогда любой эквивалентный ему элемент $b$ также обладает соответствующим свойством.

Доказательство непосредственно вытекает из определений.

Лемма 2. Если а -базисный элемент и $S$-система порождающих алгебры $\mathfrak{U}$, то а эквивалентен некоторому элементу с из $S$.

Утверждение очевидным образом вытекает из условия и импликации (1).

Лемма 3. Отношение эквивалентности между элементами сохраняется при гомоморфизме унарных алгебр, то есть если $\varphi: A \rightarrow B-$ некоторый гомоморфизм унарных алгебр А и $B, a, b \in A$ и $a \sim b$, то $a \varphi \sim b \varphi$.

Доказательство. Пусть выполнены условия леммы. Тогда $(a)=(b)$. Из включения $a \in(b)$ следует, что $a=w b$ для некоторого $w \in \Omega^{*}$, откуда $a \varphi=w(b \varphi)$ и значит, $a \varphi \in(b \varphi)$. Симметричным образом получаем, что справедливо включение $b \varphi \in(a \varphi)$, откуда $(a \varphi)=(b \varphi)$. Лемма доказана.

\section{2. Независимые системы порождающих унарной алгебры. Лемма о замене}

Пусть $\mathfrak{U}=\langle A, \Omega\rangle$ - унарная алгебра. Элементы $a, b \in A$ называются независимыми, если каждый из них не принадлежит подалгебре, порожденной другим, то есть если $a \notin(b)$ и $b \notin(a)$.

Непустое подмножество $S \subseteq A$ называется независимым, если оно либо одноэлементно, либо любые два его элемента независимы.

Лемма 4. Если элементы а и $b$ независимы, $a \sim c, b \sim d$, то элементы $c$ u $d$ также независимы.

Доказательство. Пусть $a \sim c, b \sim d$, но элементы $c$ и $d$ не являются независимыми, например, $c \in(d)$. Тогда $c \in(b)$, поскольку $d \sim b$. Следовательно, $(c) \subseteq(b)$, откуда в силу эквивалентности элементов $a$ и $c$ вытекает включение $(a) \subseteq(b)$, что противоречит независимости элементов $a$ и $b$. Лемма доказана.

Система $S$ порождающих алгебры $\mathfrak{U}$ называется независимой (НСП), если $S-$ независимое подмножество носителя этой алгебры.

Заметим, что унарная алгебра может не иметь независимой системы порождающих. K таким алгебрам относится, например, унар $\langle\mathbf{Z}, f\rangle$, где $\mathbf{Z}-$ множество целых чисел и $f(z)=z+1$ для любого $z \in \mathbf{Z}$.

Из определения следует, что любая независимая система порождающих является минимальной по включению среди всех систем порождающих. Кроме того, очевидно, что множество всех невыводимых элементов данной алгебры включается в любую систему порождающих и, в частности, в независимую.

Лемма 5. Любая независимая система порождающих унарной алгебры состоит из базисных элементов. 
Доказательство. Пусть $S-$ НСП алгебры $\mathfrak{U}$ и $a \in S$. Достаточно доказать, что для любого элемента $b$ из $\mathfrak{U}$ включение $(a) \subseteq(b)$ влечет равенство $(a)=(b)$.

Из включения $(a) \subseteq(b)$ вытекает, что $a=u b$ для некоторого $u \in \Omega^{*}$. Далее, $b=v c$ для некоторых $v \in \Omega^{*}$ и $c \in S$. Отсюда $a=u(v c)=(u v) c$.

Теперь из включений $a, c \in S$ вытекает равенство $a=c$. Это означает, что $b=v a$, то есть $(b) \subseteq(a)$. Лемма доказана.

Лемма 6. Если в независимой системе порождаюших унарной алгебры $\mathfrak{X}$ некоторые элементы заменить эквивалентными, то получится снова независимая система порождающих алгебры $\mathfrak{U}$.

Доказательство. Пусть $S-$ НСП алгебры $\mathfrak{A}, C \subseteq S$ и $D$ - множество элементов алгебры, полученное из $C$ заменой каждого элемента эквивалентным ему. Нужно доказать, что множество $S_{1}=(S \backslash C) \cup D$ также является НСП.

Из определения вытекает, что $S_{1}$ - система порождающих алгебры $\mathfrak{A}$.

Осталось показать, что $S_{1}$ независимо. Пусть $a, b \in S_{1}$ и $a \neq b$. Тогда, если $\{a, b\} \subseteq D$, то для них найдутся элементы $c_{1}, c_{2} \in C$ такие, что $a \sim c_{1}$ и $b \sim c_{2}$. Тогда $c_{1}$ и $c_{2}$ независимы, поскольку $C \subseteq S$, откуда по лемме 4 элементы $a$ и $b$ также независимы.

Наконец, пусть $a \in S \backslash C$ и $b \in D$. Тогда существует элемент $c \in C$ такой, что $b \sim c$. Поскольку $a, c \in S$, они независимы, откуда снова по лемме 4 вытекает, что $a$ и $b$ независимы. Лемма доказана.

Лемма 7 (о замене). Пусть а-базисньй элемент алгебры $\mathfrak{X} u S$ - некоторая ее независимая система порождающих. Тогда в множестве $S$ найдется элемент с такой, что множество $S_{1}=(S \backslash\{c\}) \cup\{a\}$ также будет независимой системой порождающих алгебры $\mathfrak{U}$.

Доказательство. Действительно, в силу леммы 2 найдется элемент $c \in S$ такой, что $a \sim c$. В силу леммы 6 этот элемент является искомым. Лемма доказана.

Теорема 1. Если унарная алгебра обладает хотя бы одной независимой системой порождаюших, то любые две ее независимые системы порождающих имеют одинаковую мощиность.

Доказательство. Пусть $S$ и $S_{1}$ - независимые системы порождающих унарной алгебры $\mathfrak{A}$. Фиксируем произвольный элемент $a \in S$. Ввиду леммы 5 он является базисным, откуда в силу леммы 2 найдется элемент $c \in S_{1}$ такой, что $a \sim c$. Этот элемент определяется единственным образом, поскольку любые два элемента, входящие в одну и ту же НСП произвольной унарной алгебры, не могут быть эквивалентными.

Зададим отображение $\varphi: S \rightarrow S_{1}$ по правилу $\varphi(a)=c$. Покажем, что $\varphi$ инъективно. Допустим, что $\varphi(a)=\varphi(b)$ для некоторых различных элементов $a, b \in S$. По определению отображения $\varphi$,

$$
\varphi(a) \sim a, \quad \varphi(b) \sim b,
$$

откуда следует эквивалентность $a \sim b$, что противоречит принадлежности элементов $a$, $b$ независимой системе $S$.

Далее, множество $\varphi(S)$ получается из $S$ заменой каждого элемента эквивалентным ему, поэтому в силу леммы 6 получаем, что $\left(S_{1} \backslash \varphi(S)\right) \cup S$ также является независимой системой порождающих данной алгебры. Отсюда, поскольку $S-$ НСП, следует, что $S_{1} \backslash \varphi(S)=\varnothing$, то есть отображение $\varphi$ сюръективно. Теорема доказана.

Непосредственно из доказательства теоремы вытекает следующее утверждение. 
Следствие 1. Для любых двух независимых систем порождающих унарной алгебры существует биекция одной из них на другую, при которой каждый элемент из области определения эквивалентен своему образу.

Применяя теперь леммы 2 и 5, получаем следующее утверждение.

Следствие 2. Если унарная алгебра имеет хотя бы одну независимую систему порождающих, то любая такая система представляет собой совокупность элементов, взятых по одному из каждого слоя, порожденного базисным элементом.

Поскольку множество всех невыводимых элементов унарной алгебры включается в любую независимую систему порождающих, следствие 2 можно дополнить следующим фактом.

Если унитарная алгебра имеет хотя бы одну независимую систему порождающих, то пара множеств $(J, D)$, где $J$ - множество всех невыводимых ее элементов, а $D-$ множество всех слоев, порожденных выводимыми элементами, определяется однозначно. В этом случае, пару $(\alpha, \beta)$, где $\alpha$ и $\beta$ означают соответственно мощности множеств $J$ и $D$, назовем рангом данной унарной алгебры.

Очевидно, что если унарная алгебра конечно порождена, то она имеет ранг $(\alpha, \beta)$, где числа $\alpha$ и $\beta$, составляющие его, являются целыми неотрицательными числами.

В дальнейшем будет использована следующая лемма.

Лемма 8. Пусть $\mathfrak{A}$ - конечно порожденная унарная алгебра ранга $(m, n)$. Тогда всякая ее система порождающих, содержащая $m+n$ элементов, является независимой.

Справедливость леммы тривиальным образом следует из предыдущих рассуждений.

\section{3. Коммутативные унарные алгебры. Связные и сильно связные алгебры. Свойство Хопфа}

Унарная алгебра $\mathfrak{U}=\langle A, \Omega\rangle$ называется коммутативной, если $(f g) a=(g f) a$ для любых $f, g \in \Omega$ и $a \in A$. В этом случае очевидным образом выполнено тождество $\left(w_{1} w_{2}\right) a=\left(w_{2} w_{1}\right) a$ для любых $w_{1}, w_{2} \in \Omega^{*}, a \in A$.

Непосредственной проверкой убеждаемся также, что отношение взаимной достижимости элементов на $A$ является конгруэнцией алгебры $\mathfrak{A}$, то есть

$$
(\forall a, b \in A)\left(\forall u \in \Omega^{*}\right)(a \sim b \Rightarrow u b) .
$$

Унарная алгебра называется связной, если пересечение любых двух ее однопорожденных подалгебр непусто. Это означает, что унарная алгебра $\langle A, \Omega\rangle$ является связной тогда и только тогда, когда для любых двух ее элементов $a, b \in A$ существуют $u, v \in \Omega^{*}$ такие, что $u a=v b$.

Унарная алгебра называется сильно связной, если она порождается любым своим элементом.

Следуя [3], будем говорить, что алгебра обладает свойством Хопфа, если всякий ее эпиэндоморфизм является автоморфизмом. Нетрудно проверить, что для любой коммутативной сильно связной унарной алгебры $\mathfrak{A}$ справедливо равенство End $\mathfrak{A}=$ Aut $\mathfrak{A}$. Отсюда, в частности, вытекает, что любая коммутативная сильно связная унарная алгебра обладает свойством Хопфа. Ниже будет показано, что этим свойством обладает также всякая конечно порожденная коммутативная унарная алгебра. С этой целью сначала введем некоторые термины и докажем вспомогательное утверждение. 
Напомним, что если $\varphi: A \rightarrow A-$ произвольное отображение множества $A$ в себя, то подмножество $B \subseteq A$ называется инвариантным относительно $\varphi$, если $B \varphi \subseteq B$.

Пусть $\mathfrak{U}=\langle A, \Omega\rangle-$ произвольная унарная алгебра. Подмножество $B \subseteq A$ назовем сильно связным, если любые два его элемента порождают одну и ту же подалгебру в $\mathfrak{U}$ (то есть эквивалентны между собой).

Лемма 9. Пусть $\mathfrak{U}=\langle A, \Omega\rangle-$ коммутативная унарная алгебра, $\varphi: \mathfrak{U} \rightarrow \mathfrak{U}-$ произвольный ее эндоморфизм и $B-$ сильно связное подмножество носителя $A$ этой алгебры, инвариантное относительно $\varphi$. Тогда ограничение $\varphi_{B}$ отображения $\varphi$ на В инъективно.

Доказательство. Пусть $a \varphi=b \varphi=c$ для некоторых элементов $a, b, c \in B$. По условию леммы найдутся $u, v \in \Omega^{*}$ такие, что $b=u a$ и $a=v c$. Отсюда следует, что $c=b \varphi=(u a) \varphi=u(a \varphi)=u c$, откуда $b=u a=u(v c)=v(u c)=v c=a$. Лемма доказана.

Теорема 2. Любая конечно порожденная коммутативная унарная алгебра обладает свойством Хопфа.

Доказательство. Пусть $\mathfrak{A}=\langle A, \Omega\rangle$ - конечно порожденная коммутативная унарная алгебра и $\varphi-$ некоторый ее эпиэндоморфизм. Нужно доказать, что $\varphi-$ инъекция. Очевидно, что для этого достаточно установить, что $\varphi^{k}$ является инъекцией для некоторого $k \in \mathbf{N}$.

Фиксируем какую-нибудь независимую систему порождающих $a_{1}, a_{2}, \ldots, a_{n}$. Тогда в силу сюръективности отображения $\varphi$ элементы

$$
a_{1} \varphi, a_{2} \varphi, \ldots, a_{n} \varphi
$$

образуют систему порождающих этой алгебры. Ввиду леммы 8, система (3) также независима.

Ввиду следствия 2 из теоремы 1, система (3) представляет собой совокупность элементов, взятых по одному из каждого слоя $S\left(a_{1}\right), S\left(a_{2}\right), \ldots, S\left(a_{n}\right)$. Это означает, что $\varphi$ индуцирует подстановку на множестве $\left\{S\left(a_{1}\right), s\left(a_{2}\right), \ldots, S\left(a_{n}\right)\right\}$. Следовательно, найдется число $k \in \mathbf{N}$ такое, что выполнены условия $a_{1} \varphi^{k} \in S\left(a_{1}\right), a_{2} \varphi^{k} \in S\left(a_{2}\right), \ldots, a_{n} \varphi^{k} \in S\left(a_{n}\right)$. В качестве $k$ можно взять, например, $n !$.

Таким образом, не нарушая общности, можно предположить, что

$$
a_{1} \sim a_{1} \varphi, a_{2} \sim a_{2} \varphi, \ldots, a_{n} \sim a_{n} \varphi
$$

Покажем, что из этого условия, в свою очередь, следует, что $c \sim c \varphi$ для любого $c \in \mathfrak{U}$. Действительно, пусть $c \in \mathfrak{A}$. Тогда $c=u a_{i}$ для некоторых $i \in\{1,2, \ldots, n\}$ и $u \in \Omega^{*}$. Используя теперь условия (2) и (4), получим, что $c \varphi=u\left(a_{i} \varphi\right) \sim u a_{i}=c$, то есть $c \varphi \sim c$.

Покажем, что $S(c)$ инвариантно относительно $\varphi$ для любого элемента $c \in \mathbf{A}$. Пусть $x \in S(c)$, то есть $x \sim c$. Тогда $x \varphi \sim c \varphi \sim c$ в силу леммы 3, и значит, $x \varphi \in S(c)$.

Предположим теперь, что $a \varphi=b \varphi$ для некоторых элементов $a, b \in \mathfrak{U}$. Тогда $a \sim a \varphi=b \varphi \sim b$, откуда $S(a)=S(a \varphi)=S(b \varphi)=S(b)$. Это означает, что элементы $a$ и $b$ принадлежат подмножеству $S(a)$, инвариантному относительно эндоморфизма $\varphi$. Применяя лемму 9 , из равенства $a \varphi=b \varphi$ получим, что $a=b$. Теорема доказана. 


\section{Список литературы}

1. Карташов В. К., Лемма о замене и свойство Хопфа для унарных алгебр. В сб.: Тезисы докладов Международной алгебраической конференции, посвященной 250-летию Московского универсиmema, 2004, c. 64-65.

2. Богомолов А. М., Салий В. Н., Алгебраические основы теории дискретных систем. Физматлит, Москва, 1997.

3. Нейман Х., Многообразия групп. Мир, Москва, 1969.

Статья поступила 25.04.2007. 